\title{
A Novel Radio Wave Propagation Modeling Method Using System Identification Technique over Wireless Links in East Africa
}

\author{
Solomon T. Girma ${ }^{\mathbb{D},}{ }^{1}$ Dominic B. O. Konditi, ${ }^{2}$ and Ciira Maina ${ }^{3}$ \\ ${ }^{1}$ Department of Electrical Engineering, Institute of Science, Technology and Innovation, Pan-African University, Nairobi, Kenya \\ ${ }^{2}$ Department of Electrical and Telecommunication Engineering, School of Electrical and Electronic Engineering, Faculty of \\ Engineering and Technology, Technical University of Kenya, Nairobi, Kenya \\ ${ }^{3}$ Department of Electrical and Electronics Engineering, Dedan Kimathi University of Technology, Nyeri, Kenya
}

Correspondence should be addressed to Solomon T. Girma; solomon.tshm@gmail.com

Received 21 May 2018; Revised 3 September 2018; Accepted 17 September 2018; Published 26 November 2018

Academic Editor: Stefano Selleri

Copyright (c) 2018 Solomon T. Girma et al. This is an open access article distributed under the Creative Commons Attribution License, which permits unrestricted use, distribution, and reproduction in any medium, provided the original work is properly cited.

\begin{abstract}
Transmission of a radio signal through a wireless radio channel is affected by refraction, diffraction and reflection, free space loss, object penetration, and absorption that corrupt the originally transmitted signal before radio wave arrives at a receiver antenna. Even though there are many factors affecting wireless radio channels, there are still a number of radio wave propagation models such as Okumura, Hata, free space model, and COST-231 to predict the received signal level at the receiver antenna. However, researchers in the field of radio wave propagation argue that there is no universally accepted propagation model to guarantee a universal recommendation. Thus, this research is aimed at determining the difference between the measured received signal levels and the received signal level calculated from the free space propagation model. System identification method has been proposed to determine this unknown difference. Measured received signal levels were collected from three randomly selected urban areas in Ethiopia using a computer, Nemo test tool, Actix software, Nokia phone, and GPS. The result from the simulations was validated against the received experimental signal level measurement taken in a different environment. From the simulation results, the mean square error (MSE) was $4.169 \mathrm{~dB}$, which is much smaller than the minimum acceptable MSE value of $6 \mathrm{~dB}$ for good signal propagation, and $74.76 \%$ fit to the estimation data. The results clearly showed that the proposed radio wave propagation model predicts the received signal levels at $900 \mathrm{MHz}$ and $1800 \mathrm{MHz}$ in the study region.
\end{abstract}

\section{Introduction}

Transmission of signal through a wireless radio channel is affected by path loss which mainly depends on the distance between the receiver's antenna and transmitter's antenna, antenna's characteristics, and operating frequencies [1-10]. Furthermore, the behaviors of obstructing objects in the radio channel such as walls, terrain, buildings, vegetation, and other objects have an impact on the path loss [3,11-13]. Since signal through a wireless radio channel propagates through environments where it can be reflected, scattered, and diffracted by walls, terrain, buildings, and other objects, full information of signal transmission through wireless radio channels can only be calculated by solving Maxwell's equations with boundary conditions that express the physical characteristics of these obstructing objects $[1,2,4,12]$. Since the calculation of Maxwell's equations is difficult and the necessary parameters (permeability $\mu$ and permittivity $\varepsilon$ ) are often not available, there are a number of studies in the literature [5-10] to approximate a radio wave propagation without adopting Maxwell's equations.

However, these studies were done to integrate the characteristics of the study regions and particular operating frequencies in which the systems were to be put in place. Authors in [5] had carried out an experiment on a complex building along with all L-shaped corridors in the Republic of the Union of Myanmar. For all measurements, they used a router with an $8 \mathrm{dBi}$ omnidirectional antenna and 
TP-Link at the wireless transmitter side and a laptop and inSSIDer at wireless RX. In order to develop the proposed model, the free space model was modified. The data was taken at $2.4 \mathrm{GHz}$. Authors in [7] compared Hata, Okumura, COST-231, extension of Hata model, Hata-Davidson model, and free space model with measured field data taken from FM broadcasting in North India. By analyzing the different results, the authors found that the COST-231 model had given better results. Authors in [8] investigated path loss and path loss exponent with respect to distance in the north region of India. Field measurements were taken from $50 \mathrm{dBm}$ and $70 \mathrm{dBm}$ FM radio stations at identical distances in different locations. Path loss exponents and path losses were used for comparative analysis by changing the receiver antenna height from $4 \mathrm{~m}$ to $9 \mathrm{~m}$. From their results, a receiver antenna variation from $4 \mathrm{~m}$ to $9 \mathrm{~m}$ resulted in $7 \mathrm{~dB}$ path loss and path loss exponent slightly decreased.

Authors in [9] investigated the performance evaluation of COST-231, Hata, Okumura, free space model, extension of Hata model, and Hata-Davidson model and envisaged the most suitable model for a plane area in a northern region of India. This was done by a comparative analysis of the six empirical path loss models with respect to measured data from a $50 \mathrm{dBm}$ FM radio transmitter and transmitting antenna height of $45 \mathrm{~m}$ and a $70 \mathrm{dBm}$ FM transmitter and antenna height of $100 \mathrm{~m}$ in North India. From their investigations, the Hata-Davidson model showed better results than the extension of Hata model for longer distances but the mean square error of COST231 was found to be the minimum as compared to those of other models.

Authors in [10] measured radio signals using a CDMA plot scanner, Global Positioning System (GPS), and omnidirectional antenna at $800 \mathrm{MHz}$ and $900 \mathrm{MHz}$ frequencies in Australia. The authors used measurements to develop a propagation model which they used to compare against the free space model, Okumura-Hata models, P.1546-0, P.1546-1, and P.1546-2. A result showed that P.1546-0 and P.1546-1 provide better overall prediction of path loss compared to the traditional Okumura-Hata model. The authors in [11] measured radio signals at $900 \mathrm{MHz}$ and $1800 \mathrm{MHz}$ frequencies in urban India. They used a spectrum analyzer to collect the measurements. After analyzing their data, the Okumura model showed better results in that particular study area.

Researchers in the radio wave propagation field concluded that there is no precise enough propagation model to guarantee a recommendation [10]. This is because radio propagations are affected by variations in atmospheric conditions such as temperature, pressure, and relative humidity from place to place $[1,2,4,12,13]$.

An accurate modeling of radio wave propagation is very important in a wireless network system design and analysis [6]. Most important performance metrics commonly affected by radio wave propagation models are the received signal level and cochannel interference [6]. Therefore, there is a need to have a precise propagation model owing to the fact that overprediction results in episodic outages that in turn lead to poor system availability, an increase in system latency, loss of revenue, and an underprediction result in interference on a cochannel cell [4].

This problem prompts further studies in Japan, Europe, India, and America to find a universally reliable radio wave propagation model. However, many African regions have not been adequately studied. Especially in East Africa, the closest research conducted was by [4] in Ethiopia and [14] in Sudan who investigated the effect of rainfall attenuation on microwave and millimeter wave.

This work, therefore, is aimed at finding an alternative way of modifying the free space propagation model with the help of system identification method to provide the effect of multipath propagation in our specific study region (i.e., Ethiopia).

The remainder of this paper is structured as follows: Section 2 presents a system identification method. Results and conclusion of the whole paper are presented in Section 2.1.

\section{System Identification Method}

System identification is the science that deals with converting observations of a system into mathematical models to describe the behavior of the system under test [15-17]. Inferring a mathematical model from the observations of radio wave propagation may be another method of characterization of the radio wave environment. This is because the radio environment is a generally unknown phenomenon. Therefore, it is possible to develop a mathematical model that has a good approximation of the radio environment and underlies the measured data as well as possible.

Construction of a model from a data involves three basic entities [15-17]:

(1) Input-output data

(2) A set of candidate models

(3) A rule by which candidate models can be assessed using a data

2.1. Experimental Details. The measurements were taken from Ethio telecom's network in three major towns (i.e., Hawassa, Adama, and Jimma) in Ethiopia as shown in Figure 1. The measurements were taken on $900 \mathrm{MHz}$ and $1800 \mathrm{MHz}$ frequencies.

These measurements were collected from GSM BTS with a $33 \mathrm{dBm}$ transmitter power, frequencies of $900 \mathrm{MHz}$ and $1800 \mathrm{MHz}$, a height of $35 \mathrm{~m}$, and antenna gain of $14.5 \mathrm{dBi}$. Data were collected with the help of a computer, Nemo test tool, Actix software, Nokia phone, and GPS. A Nokia phone was kept in a car with a fixed antenna height of $1.5 \mathrm{~m}$. It has an isotropic gain of $2 \mathrm{~dB}$.

The recorded data includes received signal strength (RSS), distance from the transmitter to the receiver antenna, and geographical coordinates. The measurements were taken at an interval of $5 \mathrm{~m}$. The data have been further analyzed by comparing it with the well-known free space propagation model. The difference between the measured RSS and 


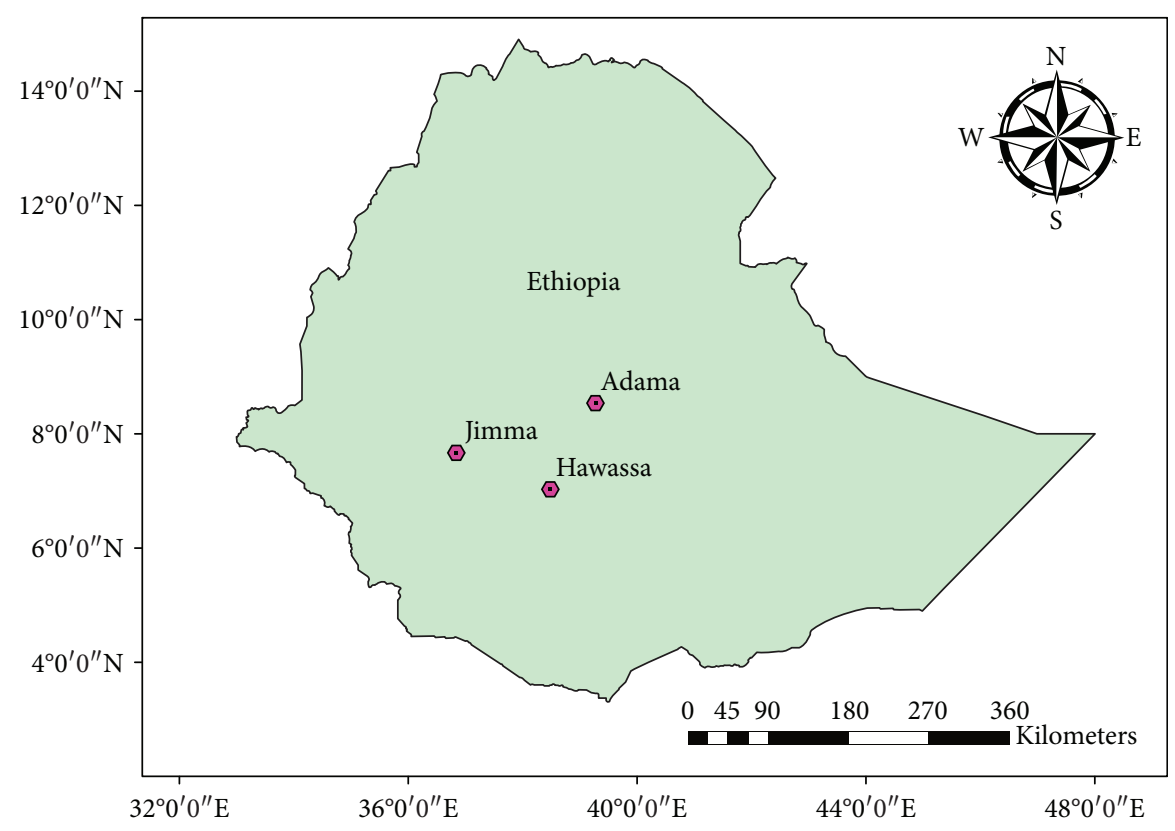

- Research sites

FIgURE 1: Study areas.

calculated RSS free space wave propagation model was used as input-output data for the system identification.

$$
\mathrm{Pr}=\mathrm{Pt}+\mathrm{Gt}+\mathrm{Gr}-\mathrm{FSl}-\text { other losses. }
$$

$\mathrm{Pr}$ is the received signal strength, $\mathrm{Pt}$ is the transmitter power, Gt and Gr are gains of the antennas, FSL is free space losses, and other losses is the overall losses of power in the air due to multipath propagation.

2.2. Standard Model Forms. There are different approaches in using transfer function methods to model unknown system parameters through system identification method. Their primary differences are on how the noise is entered into the system response $[15,16]$. The process of system identification requires that one has to choose a model structure and apply the estimation methods to determine the numerical values of the model parameters based on the system input-output data $[15,16]$. Especially if the behavior of the system is unknown, it is advised to check the mathematical structures available in the toolbox to reproduce the measured data $[15,16]$. This modeling approach is called black-box modeling $[15,16]$. On the contrary, if the first principle is known and you do not know the numerical values of some of the constants, gray modeling will help in determining the numerical values of those constants $[15,16]$.

System identification with a transfer function involves determining the transfer function between the input and output as well the noise. Let us assume the system dynamics specified by

$$
Y(z)=G(z) * U(z)+H(z) * X(z)
$$

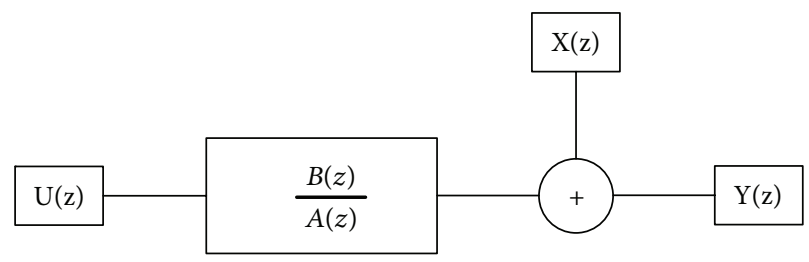

Figure 2: Transfer function of the OE model.

where $G(z)=B(z) / A(z)$ and $H(z)=C(z) / D(z), Y(z)$ is the output of the system, $U(z)$ is the input to the system, $X(z)$ is a white noise with a zero mean, $G(z)$ is a transfer function between the input and output, and $H(z)$ is a transfer function of the noise. Here, system identification will find out if the coefficients of $A, B, C$, and $D$ exist and what are the values of the coefficients.

In this paper, we look at four standard model forms that have different properties: some are easier to identify, but others are more general.

2.2.1. Output Error (OE) Model. The output error (OE) approach models the system as shown in Figure 2.

The transfer function of the output error model is given by (3). It is very good to use this model when the system noise is dominated by the white noise [9].

$$
Y(z)=X(z)+\frac{U(z) * B(z)}{A(z)},
$$

where $U(z)$ is the system input, $X(z)$ is the system disturbance, $Y(z)$ is the system output, and $B(z)$ and $A(z)$ are 


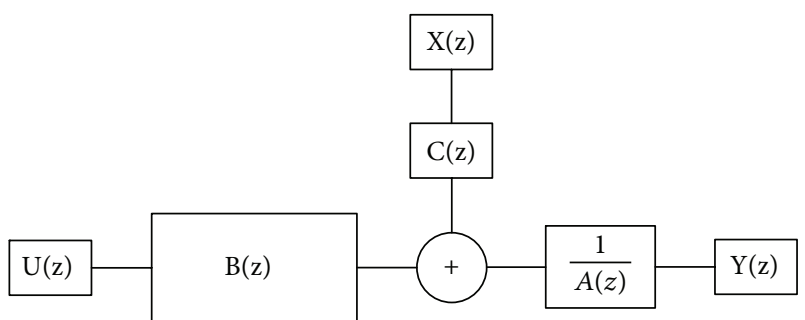

FIGURE 3: Transfer function of the ARMAX model.

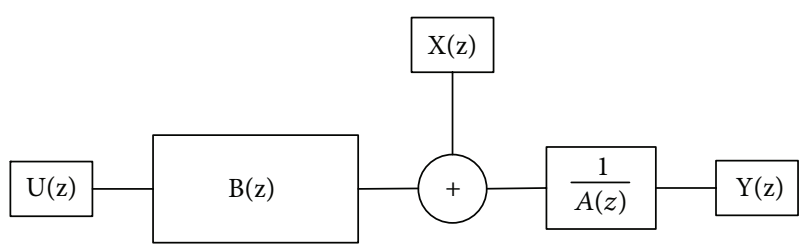

FIgUre 4: Transfer function of the ARX model.

polynomials with respect to the backward shift operator $z^{-1}$. The filters are defined as

$$
\begin{aligned}
& A(z)=1+a_{1} z^{-1}+a_{2} z^{-2}+a_{3} z^{-3}, \ldots, a_{n} z^{-n}, \\
& B(z)=b_{1} z^{-1}+b_{2} z^{-2}+b_{3} z^{-3}, \ldots, b_{n} z^{-n} .
\end{aligned}
$$

2.2.2. ARMAX Model. The autoregressive-moving-average with extra input (ARMAX) approach models the system as indicated in Figure 3.

The transfer function of ARMAX is given by (5).

$$
Y(z)=\frac{X(z) * C(z)}{A(z)}+B(z) * \frac{U(z)}{A(z)}
$$

where $U(z)$ is the system input, $X(z)$ is the system disturbance zero mean white Gaussian, $Y(z)$ is the system output, and $B(z), A(z)$, and $C(z)$ are polynomials with respect to the backward shift operator $z^{-1}$.

The filters are defined as

$$
\begin{aligned}
& A(z)=1+a_{1} z^{-1}+a_{2} z^{-2}+a_{3} z^{-3}, \ldots, a_{n} z^{-n}, \\
& B(z)=b_{1} z^{-1}+b_{2} z^{-2}+b_{3} z^{-3}, \ldots, b_{n} z^{-n}, \\
& C(z)=1+c_{1} z^{-1}+c_{2} z^{-2}+c_{3} z^{-3}, \ldots, c_{n} z^{-n} .
\end{aligned}
$$

2.2.3. ARX Model. The autoregressive with extra input (ARX) approach models the system as shown in Figure 4.

The transfer function of AMRAX is given by (7).

$$
Y(z)=\frac{X(z)}{A(z)}+B(z) * \frac{U(z)}{A(z)},
$$

where $U(z)$ is the system input, $X(z)$ is the system disturbance, $Y(z)$ is the system output, and $B(z)$ and $A(z)$ are

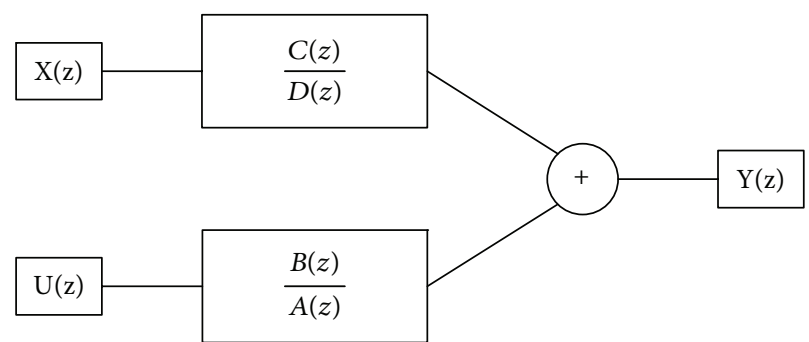

Figure 5: Transfer function of the BJ model.

polynomials with respect to the backward shift operator $z^{-1}$. The filters are defined as

$$
\begin{aligned}
& A(z)=1+a_{1} z^{-1}+a_{2} z^{-2}+a_{3} z^{-3}, \ldots, a_{n} z^{-n}, \\
& B(z)=b_{1} z^{-1}+b_{2} z^{-2}+b_{3} z^{-3}, \ldots, b_{n} z^{-n} .
\end{aligned}
$$

2.2.4. Box-Jenkins Model. The Box-Jenkins (BJ) approach models the system as shown in Figure 5.

$$
Y(z)=\frac{X(z) * C(z)}{D(z)}+B(z) * \frac{U(z)}{A(z)},
$$

where $U(z)$ is the system input, $X(z)$ is the system disturbance, $Y(z)$ is the system output, and $B(z), A(z), C(z)$, and $D(z)$ are polynomials with respect to the backward shift operator $z^{-1}$.

$$
\begin{aligned}
& A(z)=1+a_{1} z^{-1}+a_{2} z^{-2}+a_{3} z^{-3}, \ldots, a_{n} z^{-n}, \\
& B(z)=b_{1} z^{-1}+b_{2} z^{-2}+b_{3} z^{-3}, \ldots, b_{n} z^{-n}, \\
& C(z)=1+c_{1} z^{-1}+c_{2} z^{-2}+c_{3} z^{-3}, \ldots, c_{n} z^{-n}, \\
& D(z)=1+d_{1} z^{-1}+d_{2} z^{-2}+d_{3} z^{-3}, \ldots, d_{n} z^{-n} .
\end{aligned}
$$

2.3. Best Model in the Set. The assessment of the best model, after identification of the models, is determined by how the models perform well when they are applied to the same input to produce the measured output. This can be done by analyzing the mean squared errors (MSE) $[15,16]$. The best model has a lower MSE value. In the system identification, MSE can be calculated by taking the residual of a model. After picking a good performing model, based on the set criteria (autocorrelation, crosscorrelation), it remains to be seen whether the selected model is good enough for another data set. This particular test is called validation.

2.3.1. Autocorrelation Test. Residual of a proper model should be a white signal with zero means. Therefore, the residual signal can exhibit important information on the validation or invalidation of the identified model. On this ground, autocorrelation test was done to test the correlation between the residuals. In a situation of a consistent 
TABLE 1: Numerical results of the models.

\begin{tabular}{lcccc}
\hline Models & $A\left[1, a_{0}, a_{1}, a_{3} \ldots\right]$ & $B\left[1, b_{0}, b_{1}, b_{3} \ldots\right]$ & $C\left[1, c_{0}, c_{1}, c_{3} \ldots\right]$ & $D\left[1, d_{0}, d_{1}, d_{3} \ldots\right]$ \\
\hline OE & {$[1,-0.47030,-0.4928]$} & {$[0.001944,0.001752]$} & 1 & 1 \\
ARX & {$[1,-0.8927,-0.05661]$} & {$[0.03341,-0.03321]$} & 1 & 1 \\
ARMAX & {$[1,-0.3550,-0.5606]$} & {$[0.06199,-0.06167]$} & {$[1,0.5522,0.02868]$} & 1 \\
BJ & {$[1,-0.1838,-0.7775]$} & {$[0.01581,-0.01563]$} & {$[1,-0.08196,0.1028]$} & {$[1,-0.9804,0.05498]$} \\
\hline
\end{tabular}

TABle 2: Numerical results of test data.

\begin{tabular}{lccccc}
\hline Models & $\begin{array}{c}\text { Fit to } \\
\text { estimation data }\end{array}$ & FPE & $\begin{array}{c}\text { Mean } \\
\text { error }\end{array}$ & MSE & $\begin{array}{c}\text { Noise } \\
\text { variance }\end{array}$ \\
\hline OE & $31.42 \%$ & 31.11 & 0.0130 & 30.79 & 30.9670 \\
ARX & $74.76 \%$ & 4.203 & -0.0051 & 4.169 & 4.1938 \\
ARMAX & $74.89 \%$ & 4.191 & -0.0094 & 4.127 & 4.1619 \\
BJ & $74.95 \%$ & 4.154 & -0.0253 & 4.108 & 4.1251 \\
\hline
\end{tabular}

model estimate, the residual asymptotically becomes a white noise signal.

$$
\operatorname{Ree}(\tau)=\frac{1}{N} \sum_{k=0}^{N} E(t) * E(t+\tau)
$$

2.3.2. Cross-Correlation Test. Following a similar line of reasoning as that in the previous test, this test was done to check if there was a correlation between the past inputs and the residual signals. It is a requirement of a good model that the cross-correlation function between input and residuals does not go significantly outside the confidence region $[15,16]$. If not, there is an unknown parameter in the residual signals that come from the input and has not been well taken care of by the model $[15,16]$

$$
\operatorname{Reu}(\tau)=\frac{1}{N} \sum_{k=0}^{N} E(t+\tau) * U(t)
$$

2.4. Result and Discussion. Numerical results of the identified models are tabulated in Table 1. In Table 1, OE and ARX have four coefficients, whereas ARX has 6 coefficients and BJ has eight coefficients. If other tests' parameters have remained constant, $\mathrm{OE}$ and ARX models are preferred than ARMAX and BJ.

Numerical results for some of the test parameters are tabulated in Table 2. The OE model failed the MSE test and noise variance test, and it has poor estimation percentage among the other three models. ARX, ARMAX, and BJ have similar performances under the entire tests' performances, even though BJ has a little better fit to estimation data, has lower MSE, and has lower noise variance. The ARX model has slightly lower mean error over the ARMAX and BJ models. ARX, ARMAX, and BJ have satisfied the MSE test because in these models, the calculated values of MSE are $4.169,4.127$, and 4.108 , respectively, which are much smaller than the minimum acceptable MSE value of $6 \mathrm{~dB}$ for good signal propagation [8].
Residual signals have very critical information on the validation or invalidation of the developed models $[15,16]$. The best ideal model has small residual and zero mean and is uncorrelated with past samples. Therefore, the autocorrelation test was done to test the whiteness on residual of the models. At $\tau=0$, as shown in Figure 6, the autocorrelation function is 1 (this function by its definition is 1 at zero lag). However, for a model to be accepted, the autocorrelation of the residuals should be in the yellow band (i.e., the $99 \%$ confidence region) $[15,16]$. Hence, the OE model failed the whiteness test again. The autocorrelation tests of the ARX, ARMAX, and BJ models are within the recommended yellow band, and hence, the residuals of these models are white, almost zero mean, and uncorrelated.

The bottom axes in Figure 6 show the cross-correlation of the residuals with the inputs. A good model should have residuals uncorrelated with past inputs $[15,16]$. Hence, in Figure 6, it is seen that the cross-correlation values are within the yellow band for all the models.

In the summary of tests conducted, OE failed the entire test but the cross-correlation test. ARX, ARMAX, and BJ have almost similar performances. It is very difficult to pick the best model among ARX, ARMAX, and BJ on the available tests. Three of them describe the input-output data very well under the performed test with very slight differences. But, when it comes to the orders and complexity of the models, it is highly recommended to choose a model with a lower order for its simplicity $[15,16]$. On this ground, ARX edges the ARMAX and BJ models. Therefore, the ARX model was used to validate the proposed model to the measurements conducted on the different environment and to the existing wave propagation model.

The ARX model had been chosen to validate the proposed model up against the existing free space propagation model and separate measurements done to validate the proposed model. As indicated in Figure 7, the proposed model has a significant improvement over the free space propagation in Ethiopia.

2.5. Conclusion. In this paper, the validity of a system identification method to predict path loss in urban Ethiopia has been analyzed. Four standard system identification model forms, OE, ARX, ARMAX, and BJ, are used to determine the difference between measured received signal levels obtained by utilizing Nemo test tool from commercial GSM and the calculated received signal level from the free space propagation model from the same network. From the simulation results, ARX, ARMAX, and BJ have satisfied the MSE test because in these models, the calculated values of MSE are $4.169,4.127$, and 4.108 , respectively, which is much 


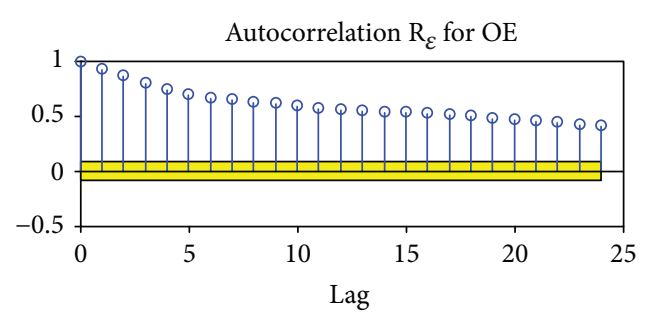

(a)

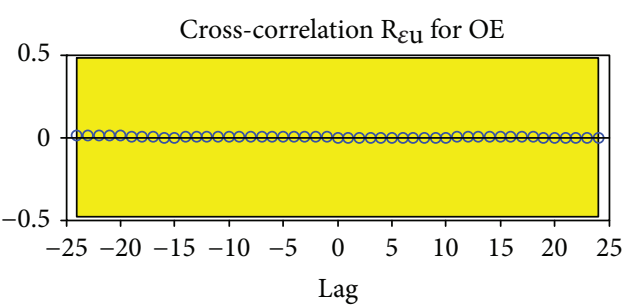

(c)

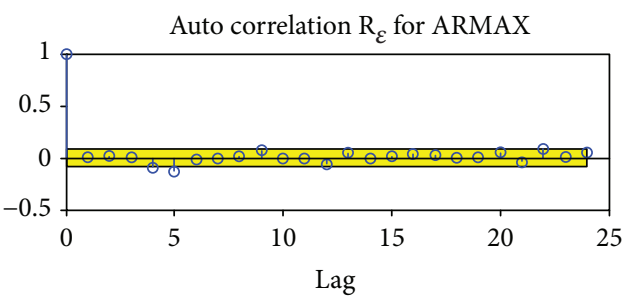

(e)

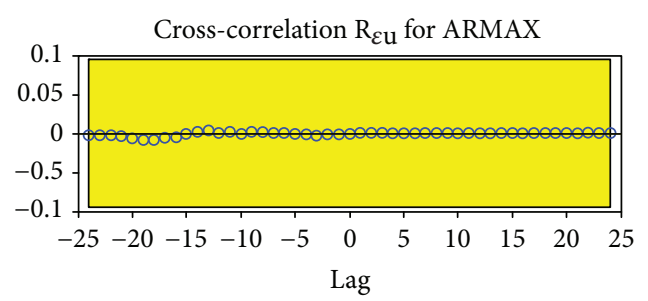

(g)

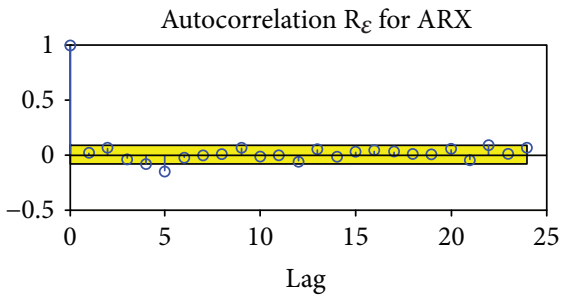

(b)

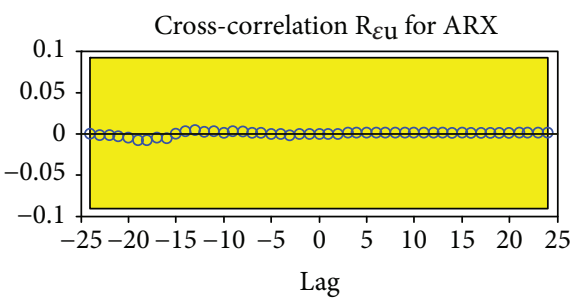

(d)

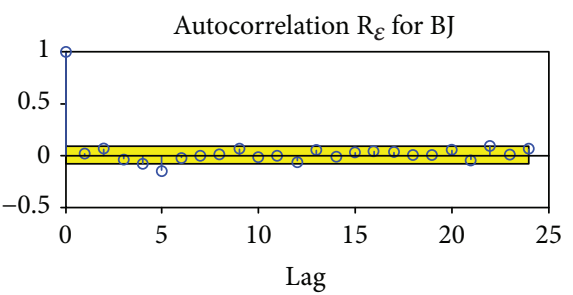

(f)

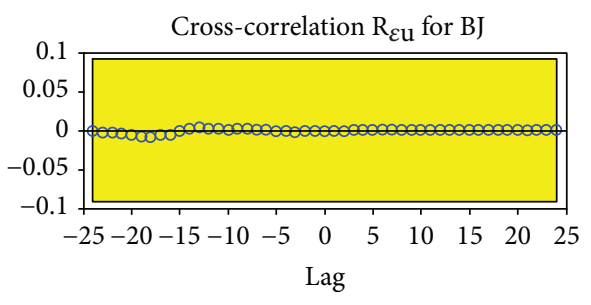

(h)

FIgURE 6: Autocorrelation and cross-correlation functions.

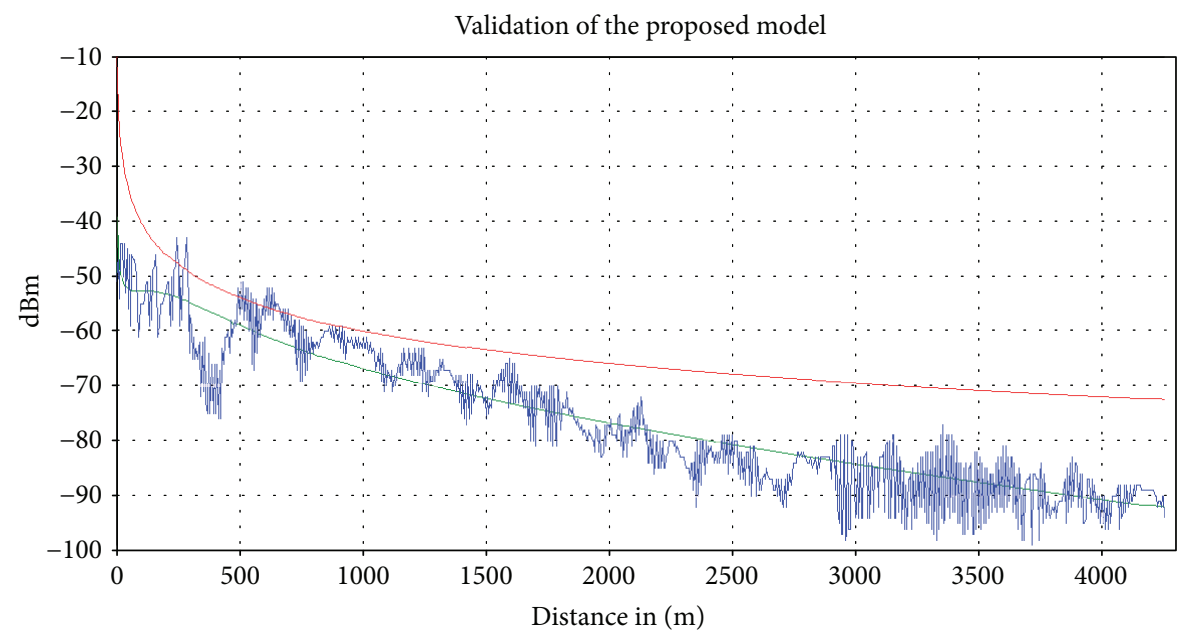

- Proposed model

- RSS measurement from the field

— Free space propagation model

FIGURE 7: Validation of the proposed model against the FSL and measurements in $\mathrm{dBm}$. 
smaller than the minimum acceptable MSE value of $6 \mathrm{~dB}$ for good signal propagation. The ARX model was used to validate the proposed model for the measurements conducted on the different environment for its simplicity. The simulation showed a promising result, that is, system identification method can be used to develop radio wave propagation mode.

\section{Data Availability}

The signal level measurement data used to support the findings of this study are available from the corresponding author upon request.

\section{Conflicts of Interest}

The authors declare that they have no conflicts of interest.

\section{Acknowledgments}

The authors want to acknowledge Eng. Gizachew Addis, Eng. Abinet Girma, and Eng. Kassahun Mokonnen who assisted us with data. This work was supported by the Institute of Science, Technology and Innovation, Pan-African University, Nairobi, Kenya.

\section{References}

[1] M. Marcus and B. Pattan, "Millimeter wave propagation: spectrum management implications," IEEE Microwave Magazine, vol. 6, no. 2, pp. 54-62, 2005.

[2] R. L. Freeman, Radio System Design for Telecommunication, IEEE, New York, NY, USA, 3rd edition, 2007.

[3] S. T. Girma and A. G. Abebe, "Mobility load balancing in cellular system with multicriteria handoff algorithm," Advances in Fuzzy Systems, vol. 2017, Article ID 2795905, 8 pages, 2017.

[4] F. D. Diba, Radio Wave Propagation Modeling under Precipitation and Clear-Air at Microwave and Millimetric Bands over Wireless Links in the Horn of Africa, University OfkwazuluNatal, 2017.

[5] T. War and A. Myint, "Development of radio wave propagation model in indoor non-line-of-sight (NLOS) scenario," International Journal of Computer, vol. 30, no. 1, 2015.

[6] S. Kurt and B. Tavli, "Path-loss modeling for wireless sensor networks: a review of models and comparative evaluations," IEEE Antennas and Propagation Magazine, vol. 59, no. 1, pp. 18-37, 2017.

[7] P. Pathania, P. Kumar, and S. B. Rana, "A modified formulation of path loss models for broadcasting applications," International Journal of Recent Technology and Engineering, vol. 3, no. 3, pp. 44-54, 2014.

[8] P. Pathania, P. Kumar, and S. B. Rana, "Performance evaluation of path loss parameters for broadcasting applications," International Journal of Research in Engineering and Technology, vol. 3, no. 4, pp. 520-528, 2014.

[9] P. Pathania, P. Kumar, and S. B. Rana, "Performance evaluation of different path loss models for broadcasting applications," American Journal of Engineering Research, vol. 3, no. 4, pp. 335-342, 2014.

[10] E. Östlin, H. Suzuki, and H. J. Zepernick, "Evaluation of the propagation model recommendation ITU-R P. 1546 for mobile services in rural Australia," IEEE Transactions on Vehicular Technology, vol. 57, no. 1, pp. 38-51, 2008.

[11] H. Haas and S. McLaughlin, "A derivation of the PDF of adjacent channel interference in a cellular system," IEEE Communications Letters, vol. 8, no. 2, pp. 102-104, 2004.

[12] A. Goldsmith, Wireless Communications, Cambridge University Press, 2005.

[13] L. MURO, Methods and Algorithms for Electromagnetic Waves Propagation in Indoor Environments, Università Degli Studi DI Napoli Federico II, 2008.

[14] S. M. Sharif, "Microwave signal attenuation due to rainfall in Sudan," The six African workshop on Wireless Use of Information, vol. 6, pp. 1-6, 2007.

[15] L. Ljung, Ljung L System Identification Theory for User. pdf, PTR Prentice Hall, Upper Saddle River, NJ, USA, 1987.

[16] L. Ljung, System Identification Toolbox ${ }^{\mathrm{тм}}$ Getting Started Guide R 2015 a How to Contact Math Works, The Math Works, 2015.

[17] E. Geerardyn, Development of User-Friendly System Identification Techniques System Identification, VUB university press, 2016. 


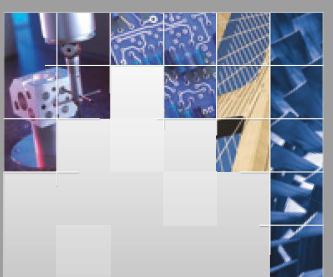

\section{Enfincering}
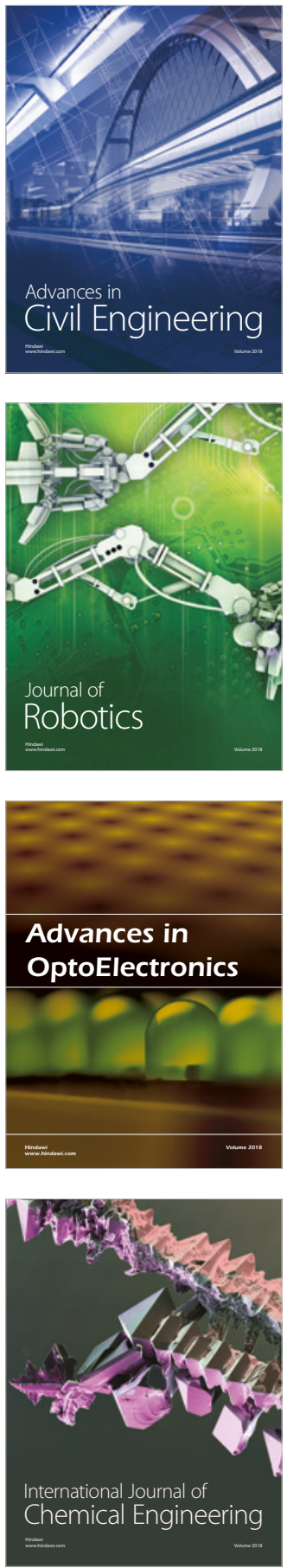

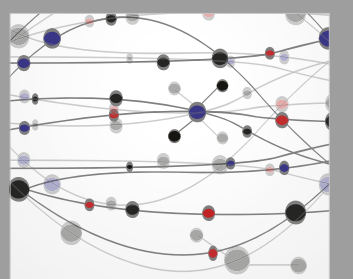

\section{Rotating \\ Machinery}

The Scientific World Journal

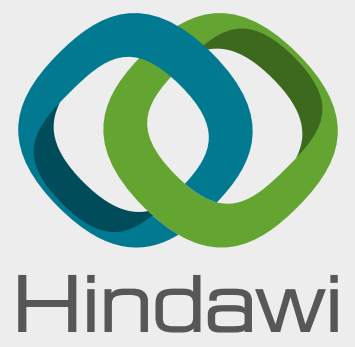

Submit your manuscripts at

www.hindawi.com
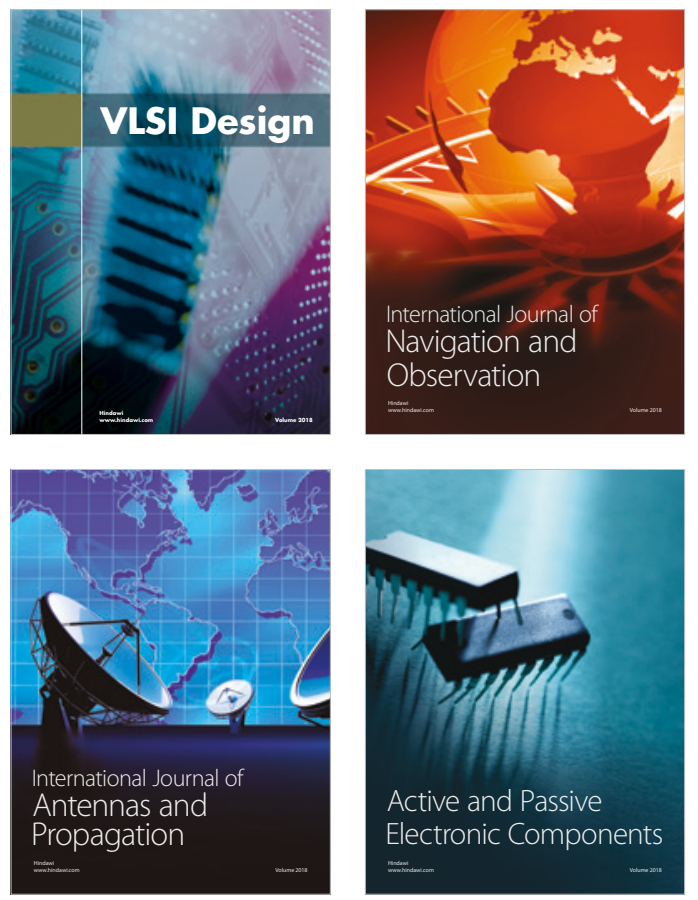
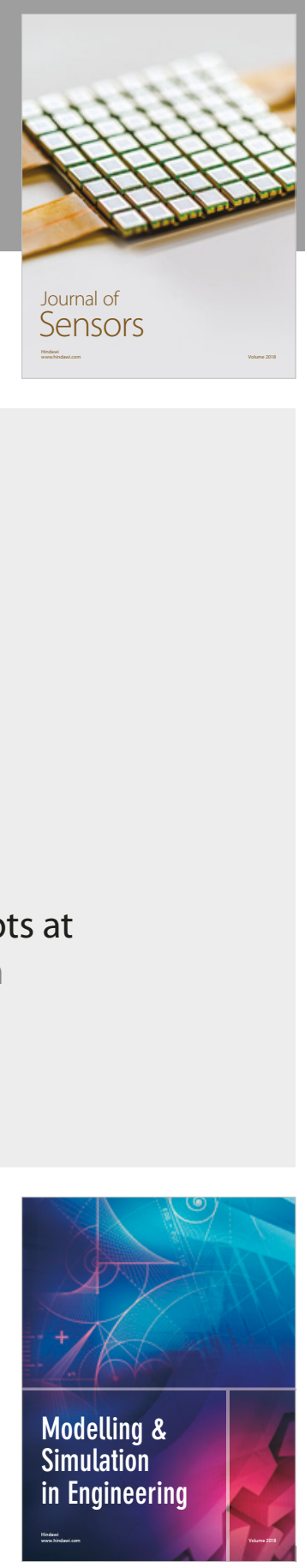

\section{Advances \\ Multimedia}
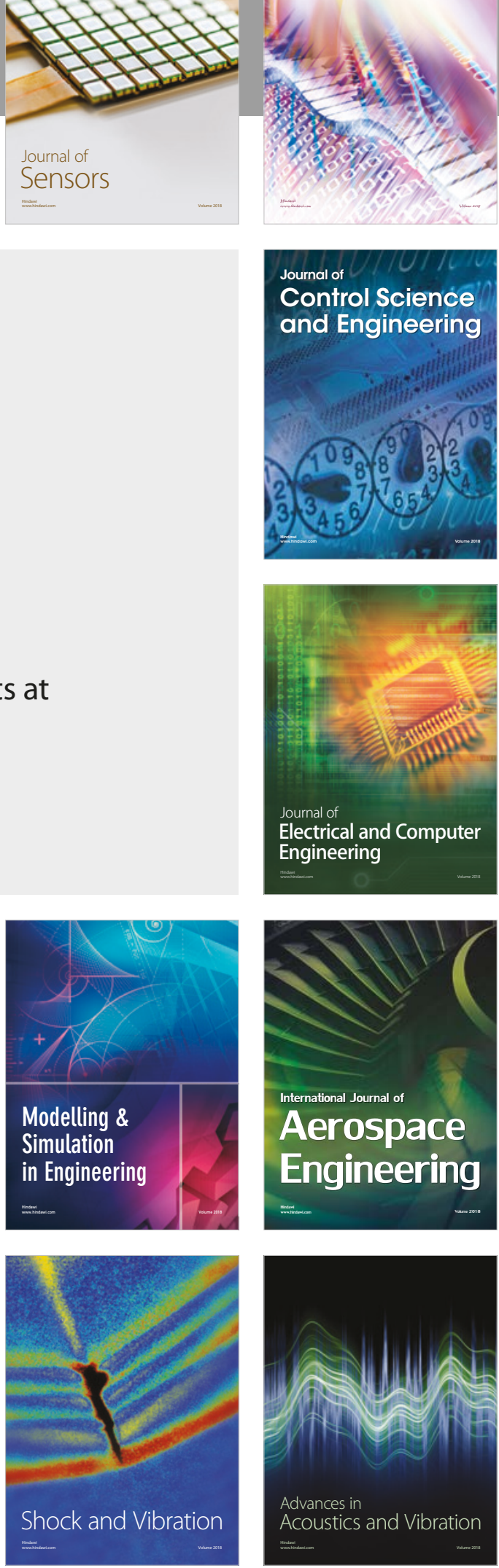